\title{
ARGESIM Benchmarks on Modelling Approaches and Simulation Implementations - Development, Classification and Basis for Simulation Education
}

\author{
Felix Breitenecker ${ }^{1 *}$, Andreas Körner ${ }^{1}$, Horst Ecker ${ }^{2}$, Nikolas Popper ${ }^{3}$, Thorsten Pawletta ${ }^{4}$ \\ ${ }^{1}$ Mathematical Modelling and Simulation Group, Inst. of Analysis and Scientific Computing, ${ }^{2}$ Inst. of Mechanics \\ and Mechatronics; TU Wien, Wiedner Hauptstrasse 8-10, 1040 Vienna, Austria; felix.breitenecker@tuwien.ac.at \\ ${ }^{3}$ dwh Simulation Services, Neustiftgasse 57-59, 1070, Vienna, Austria \\ ${ }^{4}$ Wismar Univ. of Applied Sciences, Fac. of Engineering, Research Group CEA, PF 1210, 23952 Wismar, Germany
}

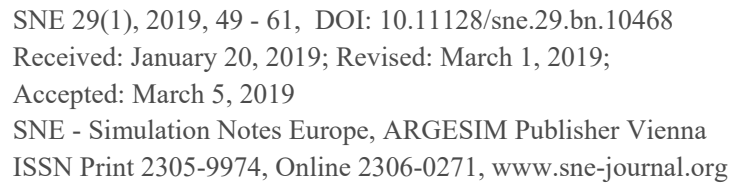

SNE 29(1), 2019, 49 - 61, DOI: 10.11128/sne.29.bn.10468 Received: January 20, 2019; Revised: March 1, 2019;

Accepted: March 5, 2019

SNE - Simulation Notes Europe, ARGESIM Publisher Vienna ISSN Print 2305-9974, Online 2306-0271, www.sne-journal.org

Abstract. ARGESIM, a non-profit association for information and publication on simulation, started in 1990 in co-operation with EUROSIM, the Federation of European Simulation Societies, the series 'ARGESIM Comparison of Simulation Software' in the journal Simulation Notes Europe (SNE). The comparisons have been developed successfully towards the 'ARGESIM Benchmarks for Modelling Approaches and Simulation Implementations', with up to now 24 benchmarks defined, and up to now 350 benchmark solutions, benchmark reports, or benchmark studies published in SNE Simulation Notes Europe.

Interestingly, it turned out, that along with the various benchmark publications in SNE, the benchmarks are used as examples, case studies, and lab work in simulation education in academia.

This contribution sketches the development of the benchmarks and introduces a new classification of the benchmarks with respect to system type, model approach, and required experiments with the model(s). In the following, main emphasis is the investigation of the benchmarks as source and basis for simulation education, together with a classification of the up to now defined benchmarks with respect to their suitability in education - from simulation methodology view, and from simulation application view. The contribution concludes with an overview table on benchmark data: definition date, number of solutions, type classification, and classification for educational use.

\section{Introduction}

The ARGESIM Benchmarks for Modelling Approaches and Simulation Implementations are a success story in the simulation community. With up to now 24 benchmarks defined, and up to now 350 benchmark solutions, benchmark reports, or benchmark studies published in SNE, the scientific membership journal of EUROSIM, the Federation of European Simulation Societies, the benchmarks publications have become a forum for information exchange on modelling approaches, simulation implementations, and features of simulation systems.

Interestingly, it turned out, that along with the various benchmark publications in SNE, the benchmarks are used as examples, case studies, and lab work in simulation education in academia - one emphasis of this contribution.

The first section lists the benchmarks defined up to now and describes the development from simulation software comparison to comparative studies on modelling approaches and simulation implementations, along with the development of the type of benchmark publications in SNE.

The second section discusses the impact of the benchmarks for simulation education generally, following the observed practice of use of the benchmarks as examples, case studies and lab work in simulation education in academia. Furthermore, the section introduces the new SNE publication type Educational Benchmark Note, for benchmark publications with primarily educational purpose and underlines the need for review of the benchmarks for educational use and classification of this educational use. The third section deals with the second emphasis of this contribution - the classification of the benchmarks with respect to system type, special modelling aspects, and required experiments. 
The fourth section inspects in detail the benchmarks with respect to education use and combines a classification for education use with the new benchmark type classification. The section finishes with an overview table on benchmark data: definition date, number of solutions, type classification, and classification for educational use.

The fifth section sketches briefly the simulation software used in benchmarks contributions, and reviews shortly the context with the benchmark development. The last section is a short Call for Benchmark Contributions.

\section{Benchmark Development}

ARGESIM, a non-profit association for information and publication on simulation, started in 1990 in co-operation with EUROSIM, the Federation of European Simulation Societies, the series ARGESIM Comparison of Simulation Software in the journal Simulation Notes Europe (SNE). The comparisons have been developed successfully towards the ARGESIM Benchmarks for Modelling Approaches and Simulation Implementations, with various publication in SNE, and publications in other journals and conference proceedings.

ARGESIM started in 1990 the series Comparison of Simulation Software with modelling and simulation tasks based on relatively simple, easily comprehensible processes. In the beginning, simulationists were invited to prepare a comparison solution to be published in SNE as one-page solution.

\subsection{Comparison and benchmark definitions}

Along with development of system simulation, also the comparisons of simulation software developed further on towards benchmarks for modelling approaches and simulation implementations.

This development can be seen in definitions and solutions published from 1990 to 2018 in 95 SNE issues: 23 definitions (some revised), and about 350 publications with solutions. The following list of comparisons and benchmarks shows also the broad variety of simulation applications:

- C01 Lithium-Cluster Dynamics, SNE 0(1), 1990

- C02 Flexible Assembly System, SNE 1(1), 1991

- C03 Generalized Class-E Amplifier, SNE 1(2), 1991

- C04 Dining Philosophers I, SNE 1(3), 1991

- C05 Two State Model, SNE 2(1), 1992

- C06 Emergency Department SNE 2(3), 1992
- C07 Constrained Pendulum, SNE 3(1), 1993

- CP1 Parallel Simulation Techniques, SNE 4(1), 1994

- C08 Canal-and-Lock System, SNE 6(1), 1996

- C09 Fuzzy Control of a Two Tank System, SNE 6(2), 1996, revised SNE 16(3), 2006

- C10 Dining Philosophers II, SNE 6(3), 1996

- C11 SCARA Robot, SNE 8(1), 1998

- C12 Collision of Spheres, SNE 9(3), 1999

- C13 Crane Crab and Embedded Control, SNE 11(1), 2001; rev. SNE 17(1), 2007

- C14 Supply Chain, SNE 11(2-3), 2001

- C15 Clearance Identification, SNE 12(2-3), 2002

- C16 Restaurant Business Dynamics, SNE 14(1), 2004

- C17 SIR Type Epidemic with CA and ODEs, SNE 14(2-3), 2004; revised SNE 25(2), (2015)

- C18 Neural Networks vs. Transfer Functions, SNE 15(1), 2005

- C19 Pollution in Groundwater Flow, SNE 15(2-3), 2005, revised SNE 16(3-4), 2006

- CP2 Parallel\& ${ }_{4}$ Distributed Simulation, SNE16(2), 2006

- C20 Complex Assembly System, SNE 21(3-4), 2011

- C21 State Events and Structural-dynamic Systems, SNE 26(2), 2016

- C22 Non-standard Queuing Policies, planned 2019

For details, see www.sne-journal.org/benchmarks/

\subsection{From comparisons to benchmarks}

In 2006, a re-organisation of the comparisons has been started [1], as the investigation of features of simulation systems went into the background, and the emphasis tended towards outline of modelling approaches and implementation techniques. The comparisons developed towards Benchmarks for Modelling Approaches and Simulation Implementations. The general changes are:

- Revised definitions: SNE is publishing revised definitions of previous comparisons, updating models and tasks in order to continue them as benchmark.

- Extended solution documentation: SNE allows two (or more) pages for solutions of classic benchmarks.

- Extended benchmarks: SNE introduces extended benchmarks, comparing modelling and simulation paradigms, or dealing with more complex models and experiments - as with benchmarks C12, C19, CP2, C20, C21, and C22.

The benchmark publications now may have different content, structure, emphasis, and length. Modellers and simulationists are invited to take the challenge to prepare, realise and submit a: 
- Benchmark Solution with concise description of model implementation and experimentation tasks (two pages SNE), or a

- Benchmark Report with sufficient detailed description of model implementation with variants and adequate experiment formulations (four to six pages SNE), or a

- Benchmark Study presenting e.g. different / alternative / comparative modelling approaches and sketching analysis variants or supplemental model experiments (six to ten pages SNE).

\section{Benchmarks and Simulation Education}

Interestingly, during some archival work in 2017 and 2018, it turned out, that along with the various benchmark publications in SNE, the benchmarks are used as examples, case studies, and lab work in simulation education in academia.

As consequence, SNE invites authors to submit their benchmark publication also with educational impact, from sketching educational aspects, until a documented teaching template for the process under investigation (see also first announcement in SNE 28(4), [1]. Benchmark reports and benchmark studies become also a SNE Educational Note:

\section{- Educational Benchmark Report}

with sufficient detailed description of model implementation with variants and adequate experiment formulations and with educational impact (four to six pages SNE), or a

\section{- Educational Benchmark Study} presenting e.g. different /alternative / comparative modelling approaches and sketching analysis variants or supplemental model experiments on an educational basis suitable for use in teaching (six to twelve pages SNE).

\subsection{Benchmarks and types of SNE Notes}

Generally, SNE publishes peer reviewed contributions on developments and trends in modelling and simulation in various areas and in application and theory, with main topics being simulation overall aspects and interdisciplinarity. Benchmark publications are seen as classical scientific publication, and categorized within the SNE publication types as Benchmark Note (BN).
As benchmark publications with educational impact may be also seen as Educational Note (EN), SNE is extending its publication type specification from 2018 on:

- Technical Note (TN) - scientific publication on specific topics in modelling and simulation, $6-10$ pages

- Short Note (SN) - recent development on specific topics, short case study, max. 6 pages

- Education Note (EN) - modelling and simulation in / for education and e-learning, 6 - 8 pages

- Software Note (SW) - specific implementation with scientific analysis, max. 6 pages

- Project Note $(\mathrm{PN})$ - Intermediate or final report or summary on a simulation project, $6-10$ pages

- Benchmark Note (BN) - Documentation of a realized ARGESIM Benchmark as Benchmark Solution, 2 pages Benchmark Report, 4 - 6 pages Benchmark Study, 6 - 10 pages

- Educational Benchmark Note (BNE)

- Documentation of a realized ARGESIM benchmark suited for educations as Educational Benchmark Report, 4 - 6 pages, or as, Educational Benchmark Study, 6 - 10 pages

- Overview Note $(\mathrm{ON})$ - State-of-the-Art report in a specific area, only upon invitation - up to 14 pages

The SNE publication type can be also seen in the header frame as picture, and within the DOI of the publication, for instance the Educational Benchmark Note [2] in SNE 28(4) ([2], DOI: 10.11128/sne.28.bne12.10452, and this contribution itself with DOI: 10.11128/sne.29.bn.10453

As another consequence of the observed educational use, the recent benchmark and the planned new benchmarks integrate educational aspects already in the definition - with equations, tasks, and background.

\section{Benchmark Classification}

There have been some attempts to classify the benchmarks and the solutions sent in. The classifications tested ranged from a rough classification of the definition to a detailed classification of the techniques presented in the solutions sent in.

From beginning on, comparisons were roughly classified as continuous comparison, or as discrete comparison. On the other side, around 2002, for the one-page comparison solutions a detailed classification for the techniques used in each task was developed and stored in a database to be accessed from the old SNE website ([3]). 
And the two-page solutions, started in 2006, requested in the last paragraph an author-defined classification of approach and software- basis.

It turned out that the development of techniques very often overrun the detailed classification based on a certain status of an implemented method, and the author-defined classifications were sometimes not really significant and meaningful. As consequence the SNE Editorial Office decided to introduce a new classification only for the benchmark definitions: i) type of the underlying dynamic system and generic modelling approach, ii) type(s) of additional modelling aspects to be considered, and iii) required experiments with the model(s).

This recent benchmark classification makes use of abbreviated keywords:

- one-letter classification key $\boldsymbol{X}$ for the general type of the dynamic system and generic modelling approach,

- two-letter classification key $\boldsymbol{Y} \boldsymbol{Y}$ for special modelling aspects to be considered or to be used alternatively,

- and three-letter keys $\boldsymbol{Z Z Z}$ for the experiments necessary or suggested for the analysis of the model(s).

While in the early days of benchmarks only either continuous or discrete benchmarks were defined, the new classification introduces five general types, given in Table 1 (multiple classifications possible).

The general classification implies a modelling approach by ODEs, by PDEs, by event description, by process description, or by algorithmic description. The classification with special modelling aspects - listed in Table 2 - describes special modelling aspects to be considered, or special modelling approaches (to be used or suggested for comparison), etc.

\begin{tabular}{l|c|l} 
Type & Abb. & Comment \\
\hline Continuous & C & ODE/PDE-type model \\
\hline Discrete & D & Model type Event, Process \\
\hline Algorithmic & A & Model type Generic Discrete \\
\hline Hybrid & H & $\begin{array}{l}\text { Continuous model with } \\
\text { discrete intervention }\end{array}$ \\
\hline $\begin{array}{l}\text { Structural- } \\
\text { dynamic }\end{array}$ & S & $\begin{array}{l}\text { State-dependent change of } \\
\text { continuous and discrete models }\end{array}$ \\
\hline
\end{tabular}

Table 1: Benchmark classification - general type with one-letter keys.

Simulation of dynamic systems generally involves time domain analysis - the basic experiments required for all benchmarks; but also, steady state, analytical or symbolic analysis may be useful. The experiment classification given in Table 3 - denotes also the more complex experiment tasks.

\begin{tabular}{l|c|l} 
Type & Key & Comment \\
\hline Stiffness & ST & Stiff System \\
\hline Spatial & SP & Additional spatial dynamics \\
\hline State Events & SE & State event description \\
\hline Implicit & IM & Implicit model description \\
\hline Time Events & TE & Time event description \\
\hline Scheduling & SC & Scheduling control in model \\
\hline Concurrency & CC & Concurrent events \\
\hline Control & CO & Model with control (tasks) \\
\hline Fuzzy & FZ & Fuzzy control or system \\
\hline Data & DA & Complex modelling data \\
\hline Physical Approach & PM & Modelica-type approaches \\
\hline AB-Models & AB & Agent-based modelling \\
\hline Transfer Function & TF & Transfer function modelling \\
\hline Neural Nets & NN & Modelling with / as NN \\
\hline SD Models & SD & System Dynamics modelling \\
\hline Cellular Automata & CA & Modelling with / as CA \\
\hline Petri Net & PN & Petri net modelling / analysis \\
\hline Compartment & CP & Compartment modelling \\
\hline
\end{tabular}

Table 2: Benchmark classification - special modelling aspects with two-letter keys.

\begin{tabular}{l|c|l} 
Type & Abb. & Comment \\
\hline Time Domain & TDA & time domain analysis \\
\hline Parameter & PAR & parameter study \\
\hline Steady State & SST & steady state analysis \\
\hline Optimization & OPT & parameters/function optimization \\
\hline Analytics & ANA & analytical analysis \\
\hline Sensitivity & SEN & sensitivity analysis \\
\hline Symbolics & SYM & Symbolical analysis \\
\hline Identification & IDT & model identification by data \\
\hline Numerics & NUM & handling of numerical problems \\
\hline Boundary & BVP & boundary value solution \\
\hline Validation & VAL & model/implementation validation \\
\hline Comparisons & CPM & model alternatives comparison o \\
\hline Statistics & STA & statistically driven experiments \\
\hline Conditions & CON & condition-dependent experiments \\
\hline Presentation & PRE & advanced result presentation \\
\hline Model Parts & MOP & model parts at experiment level \\
\hline Model Control & MOC & control of models (sequence) \\
\hline
\end{tabular}

Table 3: Benchmark classification - experiment classification with three-letter keys. 


\section{Educational Impact of the Benchmarks}

The early benchmarks (comparisons) concentrated mainly on features of simulation systems, so that an educational aspect can be mainly found in implementation. The more recent comparisons and benchmarks, and especially the extended benchmarks since 2006 investigate also modelling approaches, so that the benchmarks may suit also for educational purposes in modelling.

In the following the up to now defined comparisons and benchmarks are briefly sketched and investigated with respect to their suitability for educational purposes, which allows selection of a benchmark for certain educational use. The educational impact presents itself in modelling, in implementation, or in the application, and depends on the educational level. Therefore the SNE Editorial Office has suggested an educational classification $\mathbf{E}$ for the benchmarks in modelling $\mathbf{M}$, implementation $\mathbf{I}$ and application $\mathbf{A}$ with the rating $\mathbf{V}$ - very suitable, $\mathbf{S}$ suitable, $\mathbf{U}$ - useful, and for the educational $\boldsymbol{E}$ level $\mathbf{L}$ the suggestion beginners $\mathbf{B}$, and practitioners $\mathbf{P}$. A three-letter key, with a hyphen to distinguish from the benchmark content classification, compiles then the educational classification, e.g. EM-U, EI-V, EA-S, EL-P.

The new three-step classification: general type - special modelling aspects - types of required experiments is added to the following short presentation of benchmarks from the most recent one in 2019 the first one in 1990. The summarizing Table 4 list all benchmarks with content classification, educational classification, SNE definition, and number of contributions sent in.

\section{C22 Non-standard Queuing Policies}

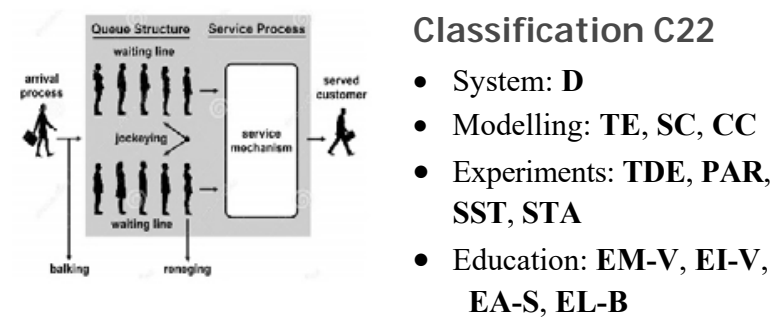

The benchmark C22 Non-standard Queuing Policies studies three non-standard queues with practical relevance: the reneging queue, where entities leave a queue after a given waiting time, the jockeying queue, where entities can switch to another shorter queue, and the classing queue, where at certain time entities with a given attribute ("class") are called to the front of the queue.
Challenges are a proper modelling approach and a suitable implementation, especially in case of concurrency, and the evaluation of the dynamic behaviour. On the one side, this benchmark is a challenge for discrete simulation systems, and on the other side this benchmark is very suited for education of beginners in modelling and implementation area.

\section{C21 State Events and Structural-dynamic Systems}

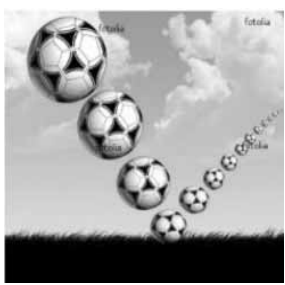

\section{C21 Classification}

- System: H, C, S

- Modelling: SE, IM, PM

- Experiments: TDA, PAR, BVP, NUM, MOC

- Education: EM-V, EI-S, EA-S, EL-B, EL-P

In benchmark C21 State Events and Structural-dynamic Systems, three case studies compare modelling and implementation of state events in dynamic systems, up to structural-dynamic systems. The first case study, the almost classical bouncing ball dynamics investigates different kinds of bounce modelling and implementation with associated events.

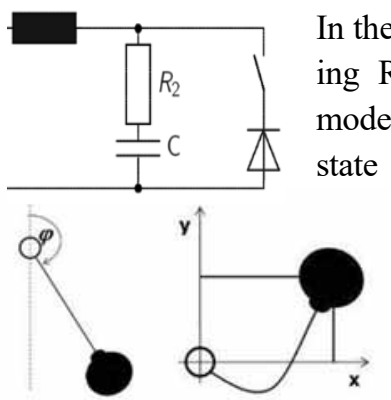

In the second case study, a switching RLC circuit, different diode models result in simple switching state events or in DAE systems which may need index reduction.

The third case study is structural-dynamic by itself: the rotating pendulum with free falling phase changes dynamics from swinging to falling (and vice versa) - switching between different degrees of freedom.

This benchmark addresses on the one side the features of simulation systems for describing and implementing hybrid and structural-dynamic systems, on the other side all three case studies are coming along with educational aspects for simulation methodology, implementation, and application. In application, the benchmark addresses mechanical engineering and electrical engineering, in methodology and implementation the benchmark deals with appropriate description and handling of hybrid and structural-dynamic systems. Depending on the case studies, the benchmark is suited for all educational levels in simulation. 


\section{C20 Complex Production System}

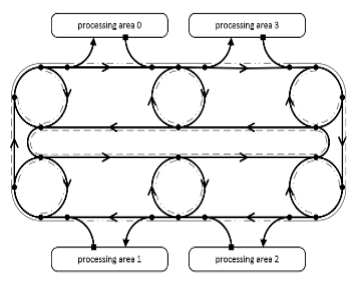

\section{C20 Classification}

- System: D

- Modelling: SC, TE, CC

- Experiments: TDA, PAR, STA

- Education: EM-U, EI-S,

EA-U, EL-P

Benchmark C20 Complex Production System is intended to be used as a foundation for analyzing manufacturing control systems, by comparing different simulation techniques and/or control algorithms regarding complexity and dynamics. The benchmark is based on two dimensions, defining a total of twelve different scenarios that differ in their complexity and dynamic behavior.

This benchmark is a challenging one for expert users in discrete process simulation and for discrete simulation systems - educational aspects can be found at implementation level.

\section{C19 Pollution in Groundwater Flow with Spatially Distributed Modelling}

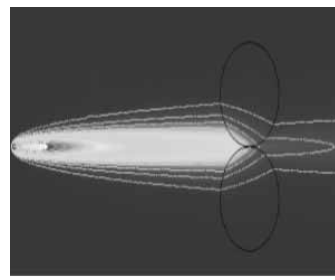

\section{C19 Classification}

- System: C

- Modelling: SP, CO, CA

- Experiments: TDA, SST, PAR, CPM

- Education: EM-V, EI-S, EA-U, EL-P

Benchmark C19 Pollution in Groundwater Flow with Spatially Distributed Modelling analyses a homogeneous ground water body with a singular pollution source and with facilities for reduction of contamination, based on the transport equation. Tasks require steady state analysis, spatial calculation of unregulated pollution spread, and modelling and control of facilities (pumps) for decontamination. The benchmark addresses quite different modelling approaches and solution techniques, from classical discretization methods via FEM to alternatives techniques like cellular automata, Monte-Carlo methods and Random Walk.

The recent revised definition emphasizes on modelling methods for distributed systems, and is a very suitable case study for introduction into instationary distributed diffusion processes - with educational aspects for comparative modelling and simulation implementation.

\section{C18 Identification of Nonlinear Dynamics - Neural Networks versus Transfer Functions

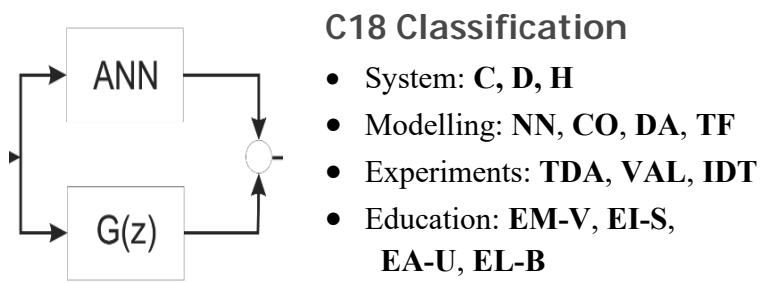

Benchmark C18 Identification of Nonlinear Dynamics - Neural Networks versus Transfer Functions studies alternative approaches for identification of the nonlinear dynamical relation between muscle force and musclebelly thickening.

Classical discrete transfer function models and as alternatives discrete transfer function models with neural net models in parallel, and extended neural net models are to be compared with respect to accuracy and efficiency. Two data sets, measured on the same muscle type, are available for identification procedures and for validation purposes (download available).

The comparative approaches invite for educational use in modelling of nonlinear dynamics and in introduction to neural nets - independent on the application area physiology. Another educational aspect is use of appropriate discrete transfer functions and error correction by neural network compensation.

\section{C17 SIR-type Epidemic with CA and ODEs}

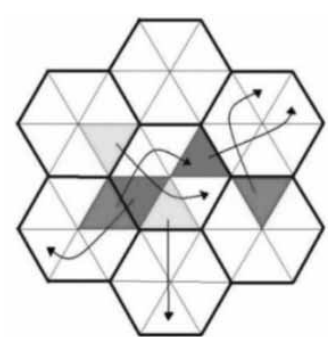

\section{C17 Classification}

- System: C, D

- Modelling: SP, CA, TE

- Experiments: TDA, PAR, IDT, CPM

- Education: EM-V, EI-S, EA-S, EL-B, EL-P

Benchmark C17 Modelling and Simulation of a SIRtype Epidemic with Cellular Automata and Ordinary Differential Equations investigates a classical population model for the spread of infection diseases (SIR model) and an inhomogeneous spatial approach using cellular automata.

The benchmark puts emphasis on identification of parameters based on an abstract time discrete conceptual model. 
Tasks include validation and analysis of identification, investigation on the impact of different spatial dynamics, and simulation scenarios for confining epidemic outbreaks that involve state-dependent interventions (vaccinations).

This benchmark is suited for educational purpose in various ways. In modelling, macroscopic and microscopic approaches can be compared, and modelling with advanced cellular automata can be studied. In implementation, discrete processes are to be formulated properly for comparative investigations. In application - spread of disease and vaccination - the benchmark is a powerful basis for testing different vaccination strategies.

\section{C16 Restaurant Business Dynamics}

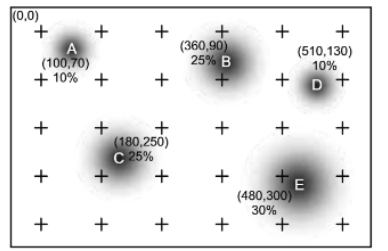

\section{C16 Classification}

- System: A, D

- Modelling: TE, SC, AB

- Experiments: TDA, PAR, OPT

- Education: EM-V, EI-V, EA-U, EL-B

Benchmark C16 Restaurant Business Dynamics addresses modelling, simulation and optimization of a discrete dynamic system. The business system under examination is restaurant operating: depending on financial results (from guests living in the neighbourhood), restaurants open branches in an adequate surrounding or close down. Strategic parameters are tax rate and structural distance to new branch restaurants - to be investigates and optimized in an adequate discrete (stochastically influenced) model (process model, agent-based model, statistical analysis, event model...).

This benchmark addresses not only the simulation community, it is an interesting calculation task for everybody, and allows various approaches, e.g. also generic algorithmic ones. Consequently, the benchmark is a basis for various education areas, also suited for spreadsheet calculation.

\section{C15 Clearance Identification}

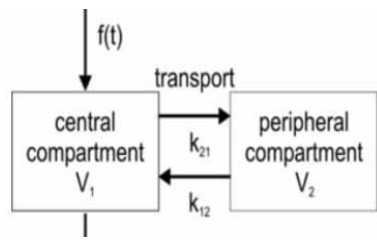

\section{C15 Classification}

- System: C

- Modelling: CP, DA

- Experiments: TDA, IDT, STA, SEN, VAL

- Education: EM-S, EI-S, EA-S, EL-B
The Benchmark C15 Clearance Identification is based on the dynamics of renal clearance. The benchmark puts emphasis on identification of a compartmental model for the clearance using real-word measurement protocols, and on statistical model parameter analysis based on artificial protocols.

This benchmark allows to study different identification approaches for a linear dynamic system, e.g. use of sensitivity functions or use of analytical solutions, and to handle artificial data protocols for extended experiments- a useful basis for teaching of both topics, independent on the physiological background.

\section{C14 Supply Chain Management}

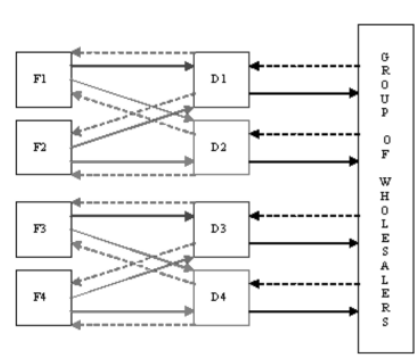

\section{C14 Classification}

- System: A, D

- Modelling: TE, AB

- Experiments: TDA, PAR, STA

- Education: EM-S, EI-S, EA-S, EL-B

Benchmark C14 Supply Chain Management is based on a simplified supply chain (four factories, four distributors, a group of wholesalers, and twelve products). The benchmark concentrates on discrete modelling of the process, especially on the order flow, and on implementation of different order strategies.

This benchmark is a classical case study for supply chain modelling, showing also the bullwhip effect dependent on the order strategies - an interesting topic for basic education in production and logistics. The implementation does not really need a simulation system, classical recursive calculations can manage all tasks, so that the benchmark is open as educational case study in many areas.

\section{C13 Crane with Complex Embedded Control}

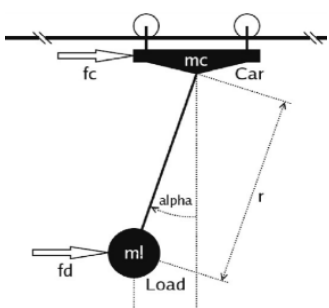

C13 Classification

- System: $\mathbf{C}, \mathbf{H}$

- Modelling: CO, SE

- Experiments: TDA, PAR, MOP, CPM, MOC, CON

- Education: EM-S, EI-S, EA-S, EL-P

Benchmark C13 Crane with Complex Embedded Control is based on modelling and digital control of a crane crab. The discrete control is designed by means of a state space observer, and by state space control.

SNE 29(1) - 3/2019 
On modelling level, setup and handling of implicit nonlinear model descriptions are investigated, and nonlinear model and linear model (linearized model) are compared. On simulation level, simulation results for nonlinear and linear dynamics without feedback control are to be compared, and two time-domain scenarios for the fully controlled nonlinear system with disturbances are investigated.

The revised definition of this benchmark underlines the educational impacts: on model level, comparison of models and integration of discrete elements are to be investigated, and on experiment level formulation of statedependent interventions. And finally, yet importantly, the benchmark is a case study for control application: digital observer control with security interventions.

\section{C12 Collision Processes in Rows of Spheres}

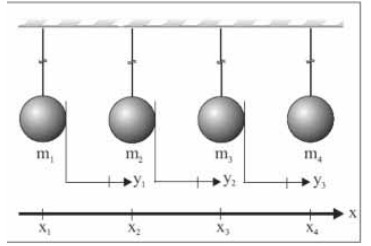

\section{C12 Classification}

- System: C, D

- Modelling: SE, TE, PM

- Experiments: TDA, NUM, PAR, CPM, STA

- Education: EM-S, EI-V, EA-V, EL-B

Benchmark C12 Collision Processes in Rows of Spheres is based on a continuous mechanical model with collision events, but mainly concentrates on discrete events within the movement of the spheres - on the collision of the spheres. The benchmark tries to analyse the phenomena of collisions, from elastic to plastic, or inelastic, resp. The tasks of the benchmark - the experiments to be performed with the model - require determination of collision sequences depending on the collision type (between elastic and inelastic), boundary value problems for initial hits, analysis of the number of collisions and of final velocities of the spheres, and stochastic analysis for stochastically modelled collisions strength. The primarily given equations are linear and can be solved analytically, so that collision times are known in advance - and an event-oriented approach is possible. But more realistic nonlinear equations require full state event handling.

This benchmark is a classical educational benchmark: demonstration of basic mechanical phenomena, various implementation possibilities because of simple linear equations, and statistically driven experiments - mainly for beginners. Interestingly, all implementations have to compensate numerical problems cause by too small slopes for determining the collisions.

\section{C11 SCARA Robot}

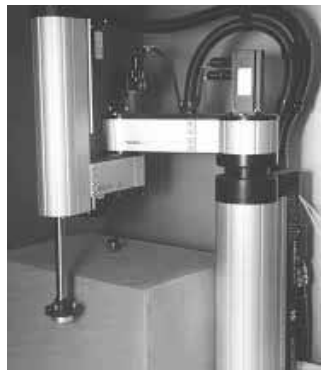

\section{C11 Classification}

- System: C, H, S

- Modelling: IM, SE, CO, PM

- Experiments: TDA, PAR, CPM, NUM

- Education: EM-V, EI-V, EA-V, EL-B, EL-P

Benchmark C12 SCARA Robot investigates movement and control of SCARA robot (Selective Compliance Assembly Robot Arm).

This robot type has two vertical revolute joints and one vertical prismatic joint. The axes of all three joints are vertical. The equations of motion follow the typical implicit form: mass matrix times accelerations equal to generalized forces. A DC-motor (ODE model with current limitations) with PD-control drives the specific movement of the robot (point-to-point control).

Challenge for implementation is the implicit model description, whereby handling requires a-priori manual resolving to an explicit state space, or numerical resolving in implementation, or use of a DAE-solver; solution may also make use of physical modelling approaches which decrease the modelling effort, but which need special care for in case of state-dependent changes.

As further challenge, the benchmark requires modelling and implementation of a collision avoidance - which requires state event handling and model change (depending on implementation).

The educational aspects of this benchmark are multifaceted: an introduction to robot modelling and control, a guideline for implementing implicit models and state event handling, and a test for the capabilities of physical modelling environments as Modelica, and some more. Interestingly, also spreadsheet calculation allows an implementation of this comparison.

\section{C10 Dining Philosophers II}

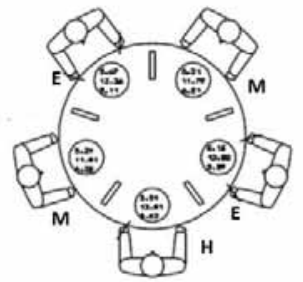

C10 Classification

- System: D, A

- Modelling: TE, SC, AB, PN

- Experiments: TDA, SST, PAR, STA, NUM, CON

- Education: EM-V, EI-V, EA-V, EL-B, EL-P 
Benchmark C10 Dining Philosophers II is a successor of the very general fourth benchmark C4 Dining Philosophers. The Dining Philosophers' problem is relatively easy to describe, but the philosophers' behaviour may cause interesting problems, including especially concurrent access and deadlock situations: Five philosophers are sitting around a large round table, each with a bowl of Chinese food in front of him. Between periods of meditation, they may start eating whenever they want to, with their bowls being filled frequently. However, there are only five chopsticks available, one each to the left of each bowl - and for eating Chinese food one needs two chopsticks.

This process offers a wide range of modelling approaches and simulation case studies - as foreseen for the original benchmark C4 Dining Philosophers. This benchmark C10 Dining Philosophers II provides better specifications for result comparisons: I) modular and / or object-oriented model descriptions, ii) investigation of simultaneous access to resources, and iii) detection of deadlocks. For this purpose, the philosophers must follow suitably settled strategies for changing the status of meditating and eating.

This definition implicitly describes also the educational impact: modelling and simulation of concurrent events, investigation of system status as deadlock or reachability. This benchmark addresses not only the simulation community, it is an interesting calculation task for everybody, and allows various approaches, e.g. also generic algorithmic ones. Consequently, the benchmark is a basis for various education areas, also suited for spreadsheet calculation.

\section{C09 Fuzzy Control of a Two Tank System}

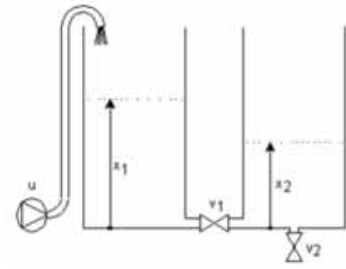

\section{C09 Classification}

- System: $\mathbf{C}, \mathbf{H}$

- Modelling: FC, CO, PM

- Experiments: TDA, CPM, NUM, PRE

- Education: EM-U, EI-S, EA-U, EL-B

Benchmark C09 Fuzzy Control of a Two Tank System is a tribute to the method of fuzzy control. A two-tank system in a specific configuration is characterized by nonlinear balancing ODEs, including characteristics of the liquid (laminar, turbulent, friction). Two fuzzy controllers (one using singletons) should be implemented as discrete systems.
The benchmark requires implementation of the fuzzy controllers and 3-D visualisation and various use of the fuzzy controllers for control of the two-tank system

From educational viewpoint, the benchmark introduces to fuzzy control and its implementation in continuous systems. The two-tank system could be described by physical modelling.

\section{C08 Canal-and-Lock System}

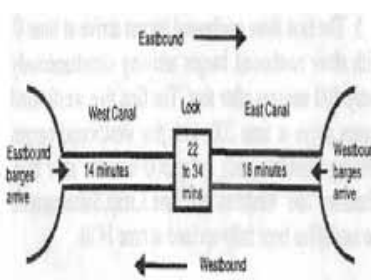

\section{c08 Classification}

- System: D, A

- Modelling: TE, SC, DA

- Experiments: TDA, STA, VAL

- Education: EM-U, EI-V, EA-U, EL-B

Benchmark C8 CanaI-and-Lock System investigates a canal-and-lock system used by barges moving from one waterway to another. The system is composed of a west canal, a lock, and an east canal. Barges can move through the system in only one direction at a time. The policy for operating the system is to let a up to maximal number barges proceed through the system in the eastbound direction, and vice versa. This ideal uniform east-to-west cycle and west-to-east cycle is 'disturbed' by less arriving barges, delayed barges, not available barges at cycle change, and differences in lock operation depending on the direction. For proper operation complex rules for the frequent exception cases must be followed.

While the systems can be modelled by simple process modelling, these complex rules are the modelling challenge in this benchmark. Task are the validation of the model (of the implementation of the complex rules) by given and solved scenarios, and statistical capacity analysis including variance reduction techniques.

This benchmark shows educational aspects for condition implementation in process modelling, and generally for conditional event modelling (as the benchmark can also be solved in a classical programming environment). On experiment level, variance reduction techniques can be trained.

\section{C07 Constrained Pendulum}

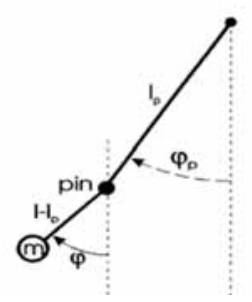

C07 Classification

- System: C, H, S

- Modelling: SE, PM

- Experiments: TDA, PAR, CPM, BVP, MOP, MOC

- Education: EM-V, EI-V, EA-S, EL-B 
Benchmark C07 Constrained Pendulum comes from classical mechanics. The physical pendulum hits a pin a state event, whereby length and angular velocity change discontinuously. Tasks are proper description of the hit (and release) event, either by switching constructs or by structural-dynamic model approaches, comparison of linear and nonlinear model dynamics, parameter studies, and boundary value calculations.

This benchmark is of high educational interest, as various techniques can be used for the necessary model change, and a proper state space transformation results in much easier hit- and release-events. There is also a clever way around the boundary value problem.

\section{C06 Emergency Department - Follow-up Treatment}

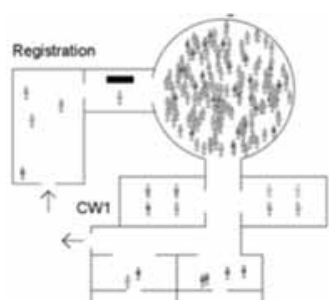

\section{C06 Classification}

- System: D

- Modelling: TE, SC, DA

- Experiments: TDA, SST, STA, CON

- Education: EM-S, EI-S, EA-S, EL-B

The Benchmark C06 Emergency Department - Followup Treatment discusses modelling approaches and strategic computer experiments for a small health care unit (post-treatment in a casualty department). The challenge for classical process-oriented modelling - with wards as stations and patients as entities - is a state-dependent change of available doctors, so that either more complex control strategies are needed, or other approaches may be meaningful. The descriptive parameters for the model statistical distributions - are based on real-world data of the health care unit.

The benchmark represents a classical introductory case study into process modelling or advanced techniques and is well suited for education in modelling and simulation.

\section{C05 Two State Model}

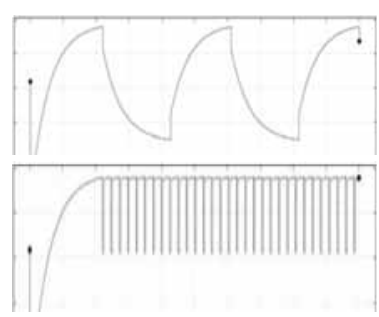

\section{C05 Classification}

- System: C, $\mathbf{H}$

- Modelling: SE

- Experiments: TDA, ANA, NUM, SYM

- Education: EM-S, EI-S, EA-U, EL-B
Benchmark C05 Two State Model deals with discontinuities in dynamic systems. While the solution itself is continuous, the derivatives may have jumps, depending on thresholds for the states. Basis is a linear stiff system with two states, where parameters in the derivative function switch depending on threshold values for the state resulting in rare switching or in high frequency switching. In any case, the switching time instants must be detected with high accuracy.

The task requires parameter variations resulting in drastically increasing number of switching, and validation of certain switching time instants with data from analytical solutions, and investigations on dependence of solutions on relative accuracy.

For education, this benchmark is suitable for getting knowledge in handling state events and mastering numerical and accuracy problems caused by stiff systems. Furthermore, numerical and analytical solutions can be compared, also for validation.

\section{C04 Dining Philosophers}

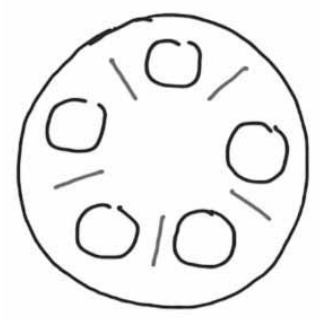

C04 Classification

- System: D, A

- Modelling: TE, CC, SC, AB, PN

- Experiments: TDA, SST, STA, PAR, ANA, SYM

- Education: EM-V, EI-V, EA-V, EL-B, EL-P

Benchmark C04 Dining Philosophers is a very general benchmark. The Dining Philosophers' problem is relatively easy to describe, but the philosophers' behaviour may cause interesting problems, including especially concurrent access and deadlock situations: Five philosophers are sitting around a large round table, each with a bowl of Chinese food in front. Between periods of meditation, they may start eating whenever they want to, with their bowls being filled frequently. However, there are only five chopsticks available, one each to the left of each bowl - and for eating Chinese food one needs two chopsticks.

This process offers a wide range of modelling approaches, analysis techniques, and simulation studies. The benchmark does not fix certain task to be performed with a model of this process - any kind of contribution with classical and alternative modelling technique, any kind of analysis technique (from numeric to symbolic), and any kind of strategy case study is welcome. This variety makes this benchmark generally very suitable for education purposes. 


\section{C03 Generalized Class-E Amplifier}

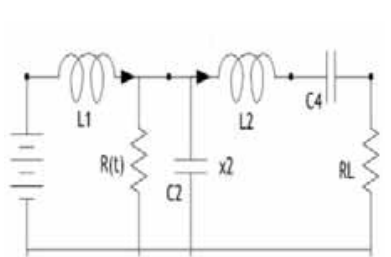

\section{C03 Classification}

- System: C, $\mathbf{H}$

- Modelling: TE, ST, PM

- Experiments: TDA, PAR, ANA, NUM, BVP

- Education: EM-U, EI-S, EA-U, EL-B

The Benchmark C03 Generalized Class-E Amplifier is based on the classical model of a class-E amplifier, consisting of two capacitors, two inductors, and two resistors mixed in serial and in parallel. Tasks are general parameter studies, steady state analysis, special parameter analysis for a switching resistor, and a boundary value problem. This benchmark offers education purposes in physical modelling for electrical engineering, and analysis techniques (numerical and analytical),

\section{C02 Flexible Assembly System}

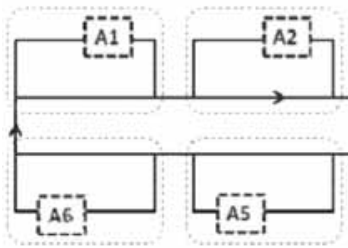

\section{Classification C02}

- System: D

- Modelling: TE, SC

- Experiments: TDE, STA

- Education: EM-U, EI-U, EA-S, EL-B

Benchmark C02 Flexible Assembly System is the first discrete comparison defined in SNE. Based on a relatively simple process model with six stations around a circular transport belt, the task is to define and combine submodels and to describe control strategies for the workflow of pallets travelling around the belt.

Originally defined for checking the features of discrete simulators for the sketched tasks, the relatively simple model is case study for introduction to discrete process modelling, or to event-based process modelling and to simulation of production systems - a suitable basis for education for simulation novices in this area.

\section{C01 Lithium-Cluster Dynamics}

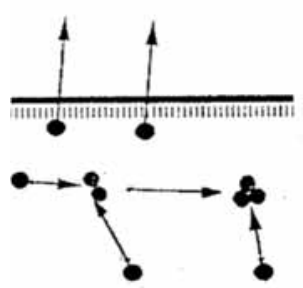

\section{C01 Classification}

- System: C

- Modelling: ST, CP

- Experiments: TDA, SST, PAR, NUM

- Education: EM-S, EI-S, EA-S, EL-B
The Benchmark C01 Lithium Cluster Dynamics, the first benchmark in SNE, is taken from solid state physics. A balance model describes formation and decay of defect aggregates in alkali halides, produced by electron bombardment. The nonlinear ODE model consists of three states (defect aggregates) and is very stiff.

Tasks are simulation of the stiff system comparing ODE solvers, a parameter study for a critical decay parameter, and steady state calculation. Emphasis of educational aspects is simulation of stiff systems, and steady state analysis.

\section{CP1 Parallel Simulation Techniques}

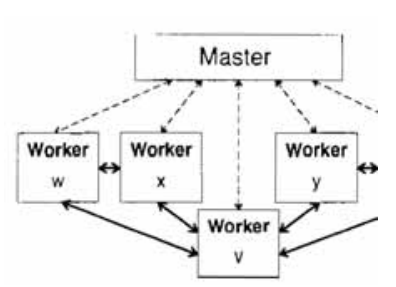

\section{Classification CP1}

- System: C

- Modelling:

- Experiments: TDE, NUM, CPM, MOP

- Education: EM-U, EI-S, EA-U, EL-B:

Benchmark CP1 Parallel Simulation Techniques deals with parallel simulation techniques. Three testcases investigate effort and benefit of parallelization of time domain for continuous models.

The first case is a parallel parameter variation of the damping factor of the classical oscillation. The ODEs for the oscillation are not coupled, the ODE solver work independently in parallel. The second case study are five strongly coupled predator-prey models. Almost all of the ten ODEs are coupled with each other, so that any kind of parallelisation of the model shows a strongly coupled structure. The third case study requires only e weak coupling: discretizing the PDE for the swinging rope by the method of lines, results in weakly couples ODEs, where one line ODE is coupled only with the neighbouring lines. This weak coupling remains also if lines are grouped together. Tasks of this benchmark is the investigation of parallelisation variants for time domain analysis, qualitatively and quantitatively in comparison with the nonparallel simulation.

This benchmark has been set up in 1994, where the simulation community was experimenting with parallelisation, at times where parallel hardware was exotic, and software for parallelisation needed special knowledge. Nowadays simulation environments make use of the parallel hardware in modern PCs automatically, so that the primarily aims of the benchmark almost have lost relevance. But this benchmark is still a proper case study for introduction into the area, so it still has impact on educational level. 


\begin{tabular}{|c|c|c|c|c|c|c|c|c|c|}
\hline \multirow{2}{*}{ Benchmark } & \multicolumn{3}{|c|}{ Type Classfication } & \multirow{2}{*}{$\begin{array}{l}\text { Definition in } \\
\text { SNE }\end{array}$} & \multirow{2}{*}{$\begin{array}{l}\dot{0} \\
\mathscr{\infty} \\
\#\end{array}$} & \multicolumn{4}{|c|}{ Education Classification } \\
\hline & $\begin{array}{l}\text { Gen. } \\
\text { Type }\end{array}$ & $\begin{array}{l}\text { Modeling } \\
\text { Aspects }\end{array}$ & Experiment & & & Mod. & Impl. & Appl. & Level \\
\hline $\begin{array}{l}\text { C22 Non-standard } \\
\text { Queuing Policies }\end{array}$ & $\mathrm{D}$ & $\begin{array}{l}\text { TE, SC, } \\
\text { CC }\end{array}$ & $\begin{array}{l}\text { TDE, PAR, SST, } \\
\text { STA }\end{array}$ & $\begin{array}{l}\text { SNE 29(3) } \\
2019 \\
\end{array}$ & & EM-V & EI-V & EA-S & EL-B \\
\hline $\begin{array}{l}\text { C21 State Events and Struc- } \\
\text { tural-dynamic Systems }\end{array}$ & $\begin{array}{c}\mathrm{H}, \\
\mathrm{C}, \mathrm{S}\end{array}$ & $\begin{array}{l}\text { SE, IM, } \\
\text { PM }\end{array}$ & $\begin{array}{l}\text { TDA, PAR, BVP, } \\
\text { NUM, MOC }\end{array}$ & $\begin{array}{l}\text { SNE 26(2), } \\
2016\end{array}$ & 3 & EM-V & EI-S & EA-S & $\begin{array}{l}\text { EL-B, } \\
\text { EL-P }\end{array}$ \\
\hline $\begin{array}{l}\text { C20 Complex Assembly } \\
\text { System }\end{array}$ & $\mathrm{D}$ & $\begin{array}{l}\text { SC, TE, } \\
\text { CC }\end{array}$ & TDA, PAR, STA & $\begin{array}{c}\text { SNE 21(3-4) } \\
2011 \\
\end{array}$ & 1 & EM-U & EI-S & EA-U & EL-P \\
\hline $\begin{array}{l}\text { C19 Pollution in } \\
\text { Groundwater Flow }\end{array}$ & $\mathrm{C}$ & $\begin{array}{l}\mathrm{SP}, \mathrm{CO} \\
\mathrm{CA}\end{array}$ & $\begin{array}{c}\text { TDA, SST, PAR, } \\
\text { CPM } \\
\end{array}$ & $\begin{array}{l}\text { SNE } 16(3-4), \\
2006, \mathrm{R}\end{array}$ & 3 & EM-V & EI-S & EA-U & EL-P \\
\hline $\begin{array}{c}\text { C18 Neural Networks vs. } \\
\text { Transfer Functions, }\end{array}$ & $\begin{array}{c}\mathrm{C}, \\
\mathrm{D}, \mathrm{H}\end{array}$ & $\begin{array}{l}\mathrm{NN}, \mathrm{CO}, \\
\mathrm{DA}, \mathrm{TF}\end{array}$ & TDA, VAL, IDT & $\begin{array}{l}\text { SNE 15(1), } \\
2005\end{array}$ & 4 & EM-V & EI-S & EA-U & EL-B \\
\hline $\begin{array}{l}\text { C17 SIR-type Epidemic } \\
\text { with CA and ODEs }\end{array}$ & C, D & $\begin{array}{l}\text { SP, CA, } \\
\text { TE }\end{array}$ & $\begin{array}{l}\text { TDA, PAR, IDT, } \\
\text { CPM }\end{array}$ & $\begin{array}{l}\text { SNE 15(2), } \\
2015, \mathrm{R}\end{array}$ & 6 & EM-V & EI-S & EA-S & $\begin{array}{l}\text { EL-B } \\
\text { EL-P }\end{array}$ \\
\hline $\begin{array}{l}\text { C16 Restaurant Business } \\
\text { Dynamics }\end{array}$ & $\mathrm{A}, \mathrm{D}$ & $\begin{array}{l}\text { TE, SC, } \\
\text { AB }\end{array}$ & TDA, PAR, OPT & $\begin{array}{l}\text { SNE 14(1), } \\
2004\end{array}$ & 10 & EM-V & EI-V & EA-U & EL-B \\
\hline $\begin{array}{l}\text { C15 Clearance } \\
\text { Identification }\end{array}$ & $\mathrm{C}$ & $\mathrm{CP}, \mathrm{DA}$ & $\begin{array}{l}\text { TDA, IDT, STA, } \\
\text { SEN, VAL }\end{array}$ & $\begin{array}{l}\text { SNE 12(2-3), } \\
2002 \\
\end{array}$ & 7 & EM-S & EI-S & EA-S & EL-B \\
\hline $\begin{array}{l}\text { C14 Supply Chain } \\
\text { Management }\end{array}$ & A, D & $\mathrm{TE}, \mathrm{AB}$ & TDA, PAR, STA & $\begin{array}{l}\text { SNE 11(2-3), } \\
2001\end{array}$ & 8 & EM-S & EI-S & EA-S & EL-B \\
\hline $\begin{array}{l}\text { C13 Crane Crab and } \\
\text { Embedded Control }\end{array}$ & $\mathrm{C}, \mathrm{H}$ & $\mathrm{CO}, \mathrm{SE}$ & $\begin{array}{l}\text { TDA, PAR, MOP, } \\
\text { CPM, MOC,CON }\end{array}$ & $\begin{array}{l}\text { SNE 17(1), } \\
2007, \mathrm{R}\end{array}$ & 12 & EM-S & EI-S & EA-S & EL-P \\
\hline C12 Collision of Spheres & $\mathrm{C}, \mathrm{D}$ & $\begin{array}{l}\text { SE, TE, } \\
\text { PM }\end{array}$ & $\begin{array}{c}\text { TDA, NUM, } \\
\text { PAR, CPM, STA }\end{array}$ & $\begin{array}{c}\text { SNE 9(3), } \\
1999 \\
\end{array}$ & 16 & EM-S & EI-V & EA-V & EL-B \\
\hline C11 SCARA Robot & $\begin{array}{c}\mathrm{C}, \\
\mathrm{H}, \mathrm{S}\end{array}$ & $\begin{array}{l}\text { IM, SE, } \\
\text { CO, PM }\end{array}$ & $\begin{array}{l}\text { TDA, PAR, } \\
\text { NUM, CPM }\end{array}$ & $\begin{array}{c}\text { SNE 8(1), } \\
1998\end{array}$ & 12 & EM-S & EI-V & EA-V & $\begin{array}{l}\text { EL-B } \\
\text { EL-P }\end{array}$ \\
\hline C10 Dining Philosophers II & $\mathrm{D}, \mathrm{A}$ & $\begin{array}{l}\text { TE, SC, } \\
\mathrm{AB}, \mathrm{PN}\end{array}$ & $\begin{array}{l}\text { TDA, SST, PAR, } \\
\text { STA, NUM, CON }\end{array}$ & $\begin{array}{c}\text { SNE 6(3), } \\
1996 \\
\end{array}$ & 23 & EM-S & EI-V & EA-V & $\begin{array}{l}\text { EL-B } \\
\text { EL-P }\end{array}$ \\
\hline $\begin{array}{c}\text { C09 Fuzzy Control of a } \\
\text { Two-Tank System }\end{array}$ & $\begin{array}{l}\text { C, } \\
\mathrm{H}, \mathrm{D}\end{array}$ & $\begin{array}{l}\mathrm{FC}, \mathrm{CO} \\
\text { PM }\end{array}$ & $\begin{array}{l}\text { TDA, CPM, } \\
\text { NUM, PRE }\end{array}$ & $\begin{array}{l}\text { SNE 6(3), } \\
2006\end{array}$ & 21 & EM-U & EI-S & EA-U & EL-B \\
\hline $\begin{array}{l}\text { C08 Canal-and-Lock } \\
\text { System }\end{array}$ & $\mathrm{D}, \mathrm{A}$ & $\begin{array}{c}\text { TE, SC, } \\
\text { DA }\end{array}$ & TDA, STA, VAL & $\begin{array}{l}\text { SNE 6(1), } \\
1996 \\
\end{array}$ & 9 & EM-U & EI-V & EA-U & EL-B \\
\hline $\begin{array}{l}\text { C07 Constrained } \\
\text { Pendulum }\end{array}$ & $\begin{array}{c}\mathrm{C}, \\
\mathrm{H}, \mathrm{S}\end{array}$ & SE, PM & $\begin{array}{l}\text { TDA, PAR, CPM, } \\
\text { BVP, MOP, MOC }\end{array}$ & $\begin{array}{c}\text { SNE 3(1), } \\
1993\end{array}$ & 43 & EM-V & EI-V & EA-S & EL-B \\
\hline $\begin{array}{c}\text { C06 Emergency } \\
\text { Department }\end{array}$ & $\mathrm{D}$ & $\begin{array}{l}\text { TE, SC, } \\
\text { DA }\end{array}$ & $\begin{array}{c}\text { TDA, SST,STA, } \\
\text { CON }\end{array}$ & $\begin{array}{l}\text { SNE 2(3), } \\
1992\end{array}$ & 20 & EM-S & EI-S & EA-S & EL-B \\
\hline C05 Two State Model & $\mathrm{C}, \mathrm{H}$ & SE & $\begin{array}{l}\text { TDA, ANA, } \\
\text { NUM, SYM }\end{array}$ & $\begin{array}{c}\text { SNE 2(1), } \\
1992\end{array}$ & 20 & EM-S & EI-S & EA-U & EL-B \\
\hline C04 Dining Philosophers I & $\mathrm{D}, \mathrm{A}$ & $\begin{array}{l}\text { TE, SC, } \\
\text { AB, PN }\end{array}$ & $\begin{array}{l}\text { TDA, SST, STA, } \\
\text { PAR, ANA, SYM }\end{array}$ & $\begin{array}{l}\text { SNE 1(3), } \\
1991\end{array}$ & 19 & EM-V & EI-V & EA-V & $\begin{array}{l}\text { EL-B } \\
\text { EL-P }\end{array}$ \\
\hline $\begin{array}{l}\text { C03 Generalized Class-E } \\
\text { Amplifier }\end{array}$ & $\mathrm{C}, \mathrm{H}$ & $\begin{array}{l}\text { TE, ST, } \\
\text { PM }\end{array}$ & $\begin{array}{l}\text { TDA, PAR, ANA, } \\
\text { NUM, BVP }\end{array}$ & $\begin{array}{c}\text { SNE 1(2), } \\
1991\end{array}$ & 31 & EM-U & EI-S & EA-U & EL-B \\
\hline $\begin{array}{l}\text { C02 Flexible Assembly } \\
\text { System }\end{array}$ & $\mathrm{D}$ & TE, SC & TDE, STA & $\begin{array}{c}\text { SNE 1(1), } \\
1991\end{array}$ & 38 & EM-U & EI-U & EA-S & EL-B \\
\hline $\begin{array}{l}\text { C01 Lithium-Cluster } \\
\text { Dynamics }\end{array}$ & $\mathrm{C}$ & ST, CP & $\begin{array}{c}\text { TDA, SST, PAR, } \\
\text { NUM } \\
\end{array}$ & $\begin{array}{c}\text { SNE 0(1), } \\
1990\end{array}$ & 38 & EM-S & EI-S & EA-S & EL-B \\
\hline $\begin{array}{l}\text { CP2 Parallel \& } \\
\text { Distributed Simulation }\end{array}$ & $\mathrm{C}, \mathrm{D}$ & & $\begin{array}{l}\text { TDE, NUM, } \\
\text { CPM, MOP }\end{array}$ & $\begin{array}{l}\text { SNE 16(2), } \\
2006\end{array}$ & 1 & EM-U & EI-S & EA-S & EL-B \\
\hline $\begin{array}{l}\text { CP1 Parallel Simulation } \\
\text { Techniques }\end{array}$ & $\mathrm{C}$ & & $\begin{array}{l}\text { TDE, NUM, } \\
\text { CPM, MOP }\end{array}$ & $\begin{array}{c}\text { SNE 4(1), } \\
1994 \\
\end{array}$ & 12 & EM-U & EI-S & EA-U & EL-B \\
\hline
\end{tabular}

Table 4: ARGESIM Benchmarks: Definition date ( $\mathrm{R}$ - revised definition), number of solutions, and classificationS:

Education E: in modelling $\mathbf{M}$ - implementation I - application A: V - very suitable , $\mathbf{S}$ - suitable, $\mathbf{U}$ - useful; Level L: beginner $\mathbf{B}$ - practitioner $\mathbf{P}$ General Type: Continuous C - Discrete D - Algorithmic A - Hybrid H - Structural-dynamic S

Modeling Aspects: Stiffness ST - Spatial SP - State Events SE - Implicit IM - Time Events TE - Scheduling SC - Concurrency CC - Control CO Fuzzy FZ - Data DA - Physical Model. PM - AB-Models AB - Transfer Function TF - Neural Nets NN - SD Models SD Cellular Automata CA - Petri Net PN - Compartment CP

Experiment: Time Domain TDA - Parameter PAR - Steady State SST - Optimization OPT - Analytics ANA - Sensitivity SEN - Symbolics SYM Identification IDT - Numerics NUM - Boundary BVP - Validation VAL - Comparisons CPM - Statistics STA - Conditions CON Presentation PRE - Model Parts MOP - Model Control MOC 


\section{CP2 Parallel \& Distributed Simulation}

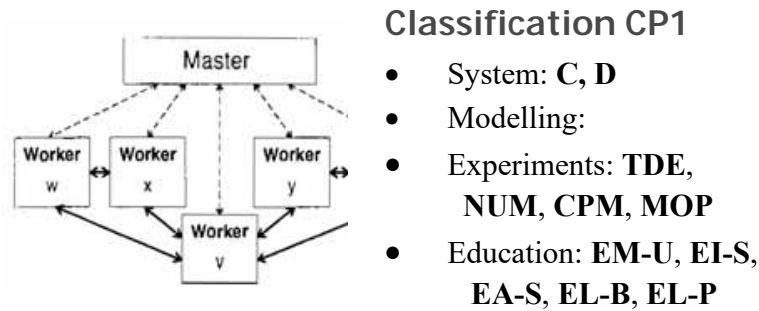

Benchmark CP2 Parallel \& Distributed Simulation updates and extends the previous ARGESIM Benchmark CP1 Parallel Simulation Techniques. Three test cases, a Monte-Carlo study, a Lattice Boltzmann Simulation, and a PDE-based Simulation investigate effort and benefit of parallelization of time domain for continuous models.

The first test case is the Monte-Carlo for the damping parameter of a classical oscillation. The ODEs for the oscillation are not coupled, the ODE solver work independently in parallel.

The second case study addresses the lattice Boltzmann method (LBM) for fluid flows, which is widespread in parallel simulation domains today. The method is derived from lattice gas cellular automata in which space, time, particle velocity and particle occupation state are all discrete. The case study is based on the famous cavity flow problem published by Hou et al in J. Comput. Phys. 118 (1995), where the behaviour of an incompressible fluid in a square enclosure, driven by a constant stream on the top boundary is examined. The task is to simulate the cavity flow with lattice size $257 \times 257$ for a number of 350.000 iterations - testing different parallelisation approaches for the flow dynamics.

The third case study is based on the PDE for a swinging string with fixed length, fixed at both ends, excited at the beginning. Discretising the space equidistant and replacing the partial differential quotient by a central difference quotient, a set of weakly coupled ODEs replaces the PDE. Also, an analytical solution (approximation) can be calculated because of the linearity of the discretized system. A classical separation approach can be used for calculating the solution (this yields with given initial and boundary conditions a solution with a Fourier series).

Nowadays simulation environments make use of the parallel hardware in modern PCs automatically, so that the primarily aims of the benchmark almost have lost relevance. But parts of this benchmark have an educational impact at modelling level, e.g. the different approaches for the swinging string PDE, and the introduction to Lattice-Boltzmann simulation.

\section{Benchmarks and Software}

In the early days, the benchmarks - at that time called Comparison of Simulation Software - primarily aimed for investigation of features of simulation software. Aim was to get benchmark contributions from as many as possible different simulation software systems. And indeed, solutions sent in presented quite different simulation software.

Along with the development of the Comparison of Simulation Software towards the Benchmarks for Modelling Approaches and Simulation Implementations also the development of simulation software underlined the shift of the emphasis of the benchmarks: more benchmarks contributions using the same usually general simulation environment, but using different approaches; and less benchmark contributions using a very specific simulation environment.

The recent benchmark contributions indicate a preference for general simulation environments and general calculation systems. So, it is not astonishing, that most frequently used software systems for benchmark contributions are MATLAB, Simulink, and AnyLogic. And very recently, contributions using Phyton - and also not surprisingly - contributions with EXCEL (with extensions) were submitted. Contributions using special simulation software are becoming a minority, and the number of contributions using a specific modelling environment (e.g. Modelica, System Dynamics, ..) or simulating in a general numerical calculation environment is growing.

\section{Benchmark Contribution - Call}

Since 2018, benchmark contributions in SNE may have different content, structure, emphasis, and length benchmark solutions, benchmark report, or benchmark study; additionally, educational aspects can be emphasis of a contribution. And furthermore, authors are free to add and modify modelling and simulation tasks of a benchmark, or to concentrate only on a part of a benchmark and extend this part. Modellers and simulationists are invited to take the challenge to prepare, realise and submit a benchmark contribution to SNE.

For details, see www.sne-journal.org/benchmarks/

\section{References}

[1] Breitenecker F, Körner A, Ecker H. Educational Use of the ARGESIM Benchmarks of Modelling Approaches and Simulation Implementations. Simulation Notes Europe SNE 28(4), 2018, 215-218. DOI: 10.11128/sne.28.bne.10453)

[2] Kolozsvari Z, Lienbacher L, et al. Time- and Event-oriented Spreadsheet Modelling of ARGESIM Benchmark C12 'Collision Processes in Rows of Spheres'. Simulation Notes Europe SNE 28(4), 2018, 205-214. DOI: 10.11128/sne.28.bne12.10452)

[3] Breitenecker F. ARGESIM Benchmarks on Modelling and Simulation: Revised Definitions, Extended Solutions, and Supplemental Information. SNE 16(3-4), 57-58, 2006. 